\title{
Blueprint for Self-replicating and Self-assembling 3D Printers
}

\author{
Mads Buch ${ }^{1,2}$ and Steen Rasmussen ${ }^{1,3}$ \\ ${ }^{1}$ FLinT Center, University of Southern Denmark \\ ${ }^{2}$ Aarhus University, Denmark \\ ${ }^{3}$ Santa Fe Institute, New Mexico, USA \\ madspbuch@gmail.com and steen@sdu.dk
}

In the 1940s Stanislaw Ulam and John Von Neumann came up with the idea of cellular Automata (CAs), which Von Neumann later used to develop a universal constructor (Neumann, 1966). The universal constructor is a pattern implemented in a CA that consists of a two parts: The universal constructor and a tape. The universal constructor reads from the tape and produces what is encoded. A universal constructor may be encoded on the tape, in which case it produces a functional copy of its own the pattern. The term "universal" in its name refers to the fact, that it itself is a universal computer, and as such it can construct all possible (computable) patterns including patters of itself. The Artificial Life community has over the years developed a variety of simple self-replicating CA patterns, e.g. see (Sayama, 2000) and references therein. The CAs, however, are not well suited for programming of arbitrary patterns. Thus, they are unpractical as universal constructors. Biological systems are also clearly examples of constructors capable of making copies of themselves, although they are not universal. They cannot be programmed to construct arbitrary systems. Significant scientific activity has been devoted to construct simple physicochemical systems capable of reproducing themselves, see e.g. (Rasmussen et al., 2008).

Since Von Neumann's proved that mathematical machines "that can make anything" do exist, such machine implementations have been a favorite item in popular scientific fiction novels and -movies. In contrast, except for a few, early, original contributions on self-replicating machines, e.g. see (Zykov et al., 2005), it's only in recent years the scientific community again has gathered around this tantalizing concept and has revamped ideas about how to implement embodied universal constructors, see e.g. (Packard et al., 2010).

To explore blueprints for embodied universal constructors is the topic of this work.

We may view two main approaches for embodied, macroscopic, universal constructors. In the one extreme we have systems where most of the necessary functional components are assembled bottom up by the internal constructor, from the available raw materials. This approach approach is exemplified by biological systems. It is difficult, however, to imagine how a macroscopic constructor, at least based on known technology, could do just that. In the other extreme, we have constructors that pick up already fully functional components and self-assemble these into new copy of themselves. A simple version of this approach is already implemented by Hod Lipson and colleagues (Zykov et al., 2005).

We choose a middle way for our constructor. Inspired by natural self-replicating systems, some components are reused and harvested from the environment while others are assembled internally bottom up. As the basis for embodiment we investigate extrusion based 3D printer technology and let the parent-printer print components which are simple, while other components either are assumed available from the environment. These components either have material- or technical constraints, which make them infeasible to print with current technology. Although the underlying code controlling a 3D extrusion printer in principle can encode any physical object and is a such universal, there are currently severe limitation on how general such physical objects can be. We will initially limit ourselves to selfreplication of and self-assembly of component.

For the extrusion based 3D printer we need material with three types of electrical properties: An isolating material, a semiconducting material and a fully conducting material. With these types of material it would be possible to build computing components (control logic) and moving components (motors). Conducting- and none-conducting plastics already exist (Leigh et al., 2012), while semiconducting plastics are not year realized, but is intensively investigated. Thus, for the time being we may assume the semiconductor plastics are provided from the environment. Additionally we need a ceramic material to e.g. to build new extrusion heads. Ceramics can easily be extruded (as clay) and later be baked to further develop more heat resistant properties.

For the self-assembler we assume a system composed of a fixed frame with a grabbing arm, which has freedom to move, grab and join objects within a certain volume. Such a contraption poses significant engineering challenges, but since various industrial robots already in use perform all the necessary actions, it should be feasible to implement such a 


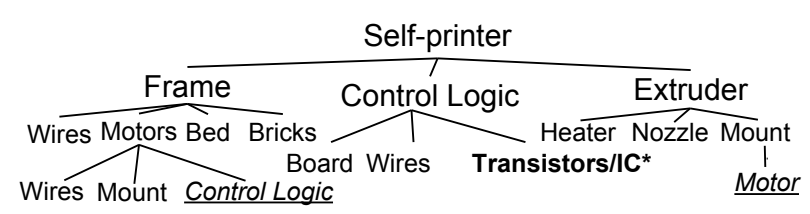

Figure 1: The component nodes and their dependencies (to the right) for the Self-printer. The leaves denotes atomic components either for producing of for retrieval from the environment. Emphasized leaves are replaced by the nonemphasized leave of the same name elsewhere.

self-assembler. It can be viewed as a generalized version of how Lipson's homogenous cubes are assembled.

A (graph) three is the natural way to express componentand process dependencies. We can therefore construct a tree expressing the components the printer needs to print, as well as a tree expressing the processes the self-assembler needs to perform, both to assemble itself and to assemble the printer.

The root node for the Self-printer is the main device itself, see Fig. 11. The Frame dependency refers to the construction that keeps all the parts together and allows for the movement of parts. The frame is printable and the implementation we use, is the Lego-printer (Møllerhøj, 2013) for the Brick-parts. Furthermore the frame depends on motors. Motors can be printed bottom up (Hawkins, 2013). The Bed (the surface that the childprinter will be printed on) should tolerate being heated, it should be flat, and it should allow the printing materials to stick. Ceramic materials may be used as a material for printing beds onto. Finally the frame needs wiring for the electronics as well as the motors. These are printable as strips of conducting plastic. Insulated wires are made by coating the conducting wires with insulator (just denoted wires in Fig. 1.). Control logic is constructed by means of $P C B$ (Printed Circuit Board). PCBs are essentially nonconducting structures that holds wires and transistors, which are easily printed (Board in Fig 1). It is not (yet) possible to print Transistors as the semiconducting material is still a technological challenge. However, if all logic is made in only transistors, the logical parts would be very large. Thus it is not practical to implement all logic in transconducting- and a non-conductingtors. Therefor we utilize IC (Integrated Circuits), which are harvested from the environment, as they cannot either (yet) be made with extrusion technology. The last thing the self-printer is dependent on is the Extruder. Both the Heater and the extrusion Nozzle are printed in a ceramic material, conducting and nonconducting respectively. The Mount is the part that holds the hot-end and feeds it with plastic. The Motor can be printed, and the rest is trivial to print. The structure requires the hotend to include heat isolation. This could be done with a ceramic layer. We may interpret Frame, Control Logic and Extruder as functionally corresponding to Container, Information and Metabolism for minimal self-replicating physic-

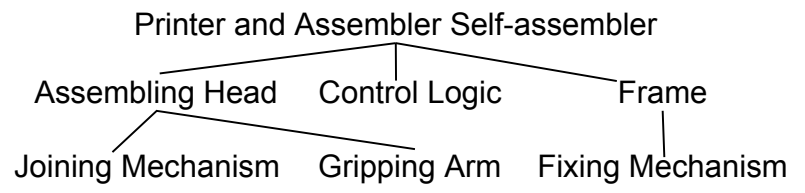

Figure 2: The dependencies in the self-assembly process. The assembling-process is specialized i.e. each individual component has a special procedure for building it (dependencies to the right).

ochemical systems (Rasmussen et al., 2008).

The Printer and Assembler Self-assembler node in Fig. 2 depends on the Frame, Control Logic and Assembly Head, which again have deeper dependencies as discussed previously. The build process is highly specialized and the components are set aside in the assembler until they are joined in their parent components. This macroscopic assembly process is very different from molecular, mainly entropy driven, self-assembly processes.

In this short presentation we focus our attention on addressing perhaps the most critical design challenges for self-replicating and self-assembling 3D printers given our laboratory experiences. We believe the component- and process dependency based design approach is appropriate for the problem at hand, and we believe the extrusion printer technology is promising as a simple first implementation technology for an early macroscopic self-replicating machine. An actual implementation of the proposed blueprint obviously raises significant practical problems. However, they should be addressable in a modern lab by a cross disciplinary team.

Hawkins, C. (2013). http://www.youtube.com/watch?v=pat638dlbs.

Leigh, S. J., Bradley, R. J., Purssell, C. P., Billson, D. R., and Hutchins, D. A. (2012). PLoS ONE, 7(11):e49365.

Møllerhøj, J. (2013). http://ing.dk/artikel/studerende-bygger-sinegen-3d-printer-i-lego-160721.

Neumann, J. V. (1966). Theory of Self-Reproducing Automata. University of Illinois Press, Champaign, IL, USA.

Packard, N., McCaskill, J., and Rasmussen, S. (2010). http://flint.sdu.dk/index.php?page=living-technology-personalfabricator.

Rasmussen, S., Bedau, M. A., Chen, L., Deamer, D., Krakauer, D. C., Packard, N. H., and Stadler, P. F., editors (2008). Protocells: bridging nonliving and living matter. the MIT press, Cambridge (Mass.).

Sayama, H. (2000). Artificial Life, 5:343-365.

Zykov, V., Mytilinaios, E., Adams, B., and Lipson, H. (2005). Nature, 435(7038):163-164. 